Participants completed a 24-question survey conducted by a single trained interviewer regarding helmet use, risk-taking behaviors, and subjective risk compensation while wearing a helmet.

Results.-In all, 938 participants (305 women, 633 men) completed the survey, $75 \%$ of whom were skiers, $25 \%$ snowboarders; 672 of 938 participants $(71 \%)$ reported always wearing a helmet. Regarding concussions, 168 of 938 (18\%) reported a PC from skiing or snowboarding; of those, $40 \%$ were snowboarders. Among participants without terrain park use, $23 \%$ of snowboarders versus $10 \%$ of skiers reported PC $\left(\chi^{2}[1, n=596]=11.22, P=.001\right)$. Among participants who spent at least some time in a terrain park, $35 \%$ of snowboarders versus $23 \%$ of skiers reported PC $\left(\chi^{2}[1, n=342]=5.83, P=\right.$ .016). Only $5 \%$ of those who reported a PC never wear a helmet. When comparing participants with PC and those without, participants with PC were more likely to subjectively compensate risk-taking behavior when wearing a helmet (odds ratio $1.94,95 \% \mathrm{CI}$ : 0.93 to $0.96, P=.001$ ). However, subjective risk compensation did not correlate with answers to follow-up questions designed to specify risk compensation.

Conclusions. - Our results suggest that, although persons with PC also report subjective risk compensation while wearing a helmet, this may affect terrain park use but may not affect their choices of terrain outside of the terrain park.

\section{Epidemiology of the Pennsic Wars 2007-2013: A Medieval Mass Gathering Event}

Philip S. Nawrocki, MD ${ }^{1}$; Peter Roolf, BSc, CCEMT-P'; Morgan Garvin, $\mathrm{MD}^{2}$; John O'Neill, $\mathrm{MD}^{1}$

${ }^{1}$ Allegheny General Hospital, Pittsburgh, PA,

${ }^{2}$ Western Pennsylvania Hospital, Pittsburgh, PA

Objective.-The Pennsic War is an annual 2-week event involving $>10,000$ participants of all ages who gather in western Pennsylvania to camp and to recreate medieval events, including full-scale battles with medieval weaponry. There is 24-hour, 7 days a week emergency medical services coverage, with a physician present during daylight hours. The closest community hospital is 30 minutes by ambulance. The objective of this study is to examine the epidemiology of an outdoor mass gathering event to as a lesson for future preparedness and response to medical emergencies at similar events.

Methods. - This is a retrospective cross-sectional chart review of patients who registered at the first aid tent during the 2007-2013 Pennsic Wars. Inclusion criteria included any patient who had data recorded into the triage log. Missing data from incomplete entries were categorized as unknown. Variables included age, sex, chief complaint, interventions and medications provided, and disposition. Data were abstracted from these triage logs by a single researcher for statistical analysis.

Results.- There were 1141 recorded visits during the 7-year study period. Dermatologic complaints were the most frequent (35\%) and commonly reported as rash, insect bite, or soft-tissue wound. Musculoskeletal (16.9\%), neurologic (10\%), and gastrointestinal $(7.3 \%)$ complaints were the next most common. The most common interventions recorded were bandaging/splinting, cardiac monitoring/ electrocardiogram, and wound closure. The most common medications dispensed included normal saline $(n=131)$, Zofran $(n=66)$, and Benadryl $(n=57)$. Most patients, $86.2 \%$, were treated and released, whereas $4.2 \%$ were evacuated to an emergency department (9\% missing data).

Conclusions. - Most presentations were not life-threatening and required only basic wound care or therapeutic measures. However, there were cases of long-bone fracture, spine injury, and overdose that required intubation. The wilderness medical provider should have a definitive stabilization and evacuation plan for the infrequent but true emergencies. Understanding the incidence and distribution of medical emergencies also aids future planning and preparation of wilderness endeavors.

\section{Bridging the Gap: Introducing Undergraduate Stu- dents to Wilderness and Emergency Medicine}

Katie E. Joy, ${ }^{1,2}$; Elaine M. Reno, MD ${ }^{1}$; Bonnie Kaplan, $\mathrm{MD}^{1}$; Todd Miner, EdD ${ }^{1}$; Jay M. Lemery, $\mathrm{MD}^{1}$

${ }^{1}$ University of Colorado School of Medicine, Aurora, $\mathrm{CO},{ }^{2}$ State University of New York at Buffalo, Amherst, NY
Objective.-Premedical-profession students across the country are a growing demographic for participation in wilderness and outdoor education activities and constitute a significant portion of the demand for free-standing wilderness medicine (WM) courses (eg, first aid, first responder). Yet, these courses often do not include education on how to pursue career opportunities in the health professions. To bridge this gap, we designed a 2-week course at the CU School of Medicine focusing on both basic backcountry medical training and exposure to and mentorship in health careers. The course had 2 major aims: first, students learned basic WM care, which included patient assessment, evaluation, stabilization, and management; second, participants gained meaningful exposure to core medical lectures, hands-on scenarios, ultrasonography, physician shadowing, admissions processes, and discussions on a wide range of healthcare-related careers.

Methods.-Surveys were distributed daily to evaluate topic relevancy, presentation quality, and overall enjoyment of material. Evaluations were distributed after the course to gauge how much students learned about emergency medicine (EM), WM, and various medical professions. Finally, students recorded their most liked and disliked areas of the course.

Results.-On average, 30 students attended each course. They reported significant gains in knowledge related to EM (9.5 of 10) and WM (9.6 of 10). In addition, they reported gaining understanding about the practice and profession of medicine (8.4 of 10). Interestingly, students rated lecture-based and hands-on activities equally, but noted that shadowing emergency medical services and emergency physicians were particularly valuable experiences.

Conclusions. - This novel educational approach influences premedical students through instruction in both hospital medicine and WM while simultaneously shaping their outlook on and ability to pursue a career in medicine. The success of these courses demonstrates a strong market demand for premedical education, portending a significant growth area for WM education as a whole.

\section{The Effect of Helmet Cameras on Risk-Taking Behavior Among Mountain Bikers}

Lauren M. Cantwell ${ }^{1}$; Meredith Ray, MPH PhD²;

Timothy J. Fortuna, $\mathrm{DO}^{3}$

${ }^{1}$ Virginia Tech Carilion School of Medicine, Roanoke, VA, ${ }^{2}$ University of Memphis, Memphis, TN, ${ }^{3}$ Department of Emergency Medicine,

Virginia Tech Carilion School of Medicine, Roanoke, VA

Objective.-The use of helmet cameras by outdoor and adventure sport enthusiasts has increased drastically. Whether their use influences the behavior of the users has not been studied. This study aimed to examine the effects of helmet camera use on mountain bikers' behavior.

Methods. - This is a prospective experimental study involving 35 local mountain bikers. Subjects rode a beginner-level course (a loop with multiple avoidable obstacles) 3 times. For the first ride, the subjects wore no camera; for the second and third rides, they wore a helmet camera. During the rides wearing the camera, subjects were told the camera was off for 1 ride and on for the other. Subjects were randomly assigned the ride during which they were told the camera was on. However, the camera filmed both rides. Data were collected from helmet camera video footage of the second and third rides and from surveys. All statistical analysis was performed using R GUI, and all statistical tests were conducted at the $\alpha=0.05$ level.

Results.-Parametric paired $t$ tests suggest a statistical difference in the time to complete the course between riders using a helmet camera and riders not using a helmet camera. The average time to complete the course for all riders was 224.50 (33.23) seconds while using the helmet camera, and 230.60 (34.35) seconds while not using the helmet camera. Twenty riders attempted the same number of obstacles, 6 attempted fewer obstacles, and 8 rode more obstacles while wearing the helmet camera compared with those not using a camera. No statistical differences were detected in the number of obstacles attempted across all riders or across different ability levels.

Conclusions.- On average, subjects using a helmet camera completed the course faster than subjects without a helmet camera but were no more likely to attempt more technical features of the course. Overall, these results do not show a significant increase in risk-taking behavior among subjects using a helmet camera. 